\title{
Calcipotriol plus betamethasone dipropionate foam is effective in patients with moderate-to-severe psoriasis: post-hoc analysis of the PSO-ABLE study
}

Carle Paul, ${ }^{1}$ Craig Leonardi, ${ }^{2}$ Alan Menter, ${ }^{3}$ Kristian Reich, ${ }^{4}$ Linda Stein Gold, ${ }^{5}$ Richard B Warren, ${ }^{6}$ Anders Moller, ${ }^{7}$ Mark Lebwoh ${ }^{8}$

'Paul Sabatier University and Larrey Hospital, Toulouse, France; ${ }^{2}$ St Louis University School of Medicine, St Louis, MO, USA; ${ }^{3 B a y l o r}$ University Medical Center, Dallas, TX, USA; ${ }^{4}$ Dermatologikum Hamburg and SCIderm GmbH, Hamburg, Germany, ${ }^{5}$ Henry Ford Health System, Detroit, MI, USA; ' ${ }^{D}$ ermatology Centre, University of Manchester, Manchester, UK; ${ }^{7} L E O$ Pharma AVS, Ballerup, Denmark, ${ }^{8} / c a h n$ School of Medicine at Mount Sinai, New York, NY, USA

\section{Introduction}

Most guidelines recommend that mild-to-moderate psoriasis be treated with topical therapies. ${ }^{12}$ Use of topical therapies in severe/extensive psoriasis is no generally recommended

- Ointment and gel formulations of fixed combination calcipotriol $50 \mathrm{\mu g} / \mathrm{g}$ (Cal) plus betamethasone $0.5 \mathrm{mg} / \mathrm{g}$ (BD) are established first-line topical treatments. ${ }^{3}$ A foam formulation has been developed with the aim of enhancing adherence and increasing the therapeufic options avallable - Studies with Cal/BD foam have demonstrated greater in vitro drug penetration and a greater antipsoriatic effect over 4 weeks of treatment than Cal/BD ointment and vehicle, with a comparable tolerablity profile The Phase III PSO-ABLE study (NCT02132936) in patients with mild-to-severe week 4 compared with Cal/BD gel at week 8 (based on the recommended

-This analysis from PSO-ABLE assesses the efficacy of Cal/BD foam and gel in the subgroup of patients with moderate-to-severe psoriasis

\section{Materials and methods}

\section{PSO-ABLE STUDY DESIG}

Paspective, minticentre, mestigator-bilinded

Call BD

\section{PATIENTS}

- Aged $\geq 18$ years with mild-to-severe psoriasis according to the physician's (S) Psoriasis Area and Severity Index (mPASI) of $\geq 2$

- For inclusion in this subgroup analysis, a patient was required to have "moderate-to-severe' psoriasis based on the "Rule of Tens'",

BSA affected $\geq 10 \%$ or mPASI score > 10 or Dermatology Life Quality Index (DLOl) score $>10$

ASSESSMENTS AND ENDPOINT

-Eficacy was assessed at weeks 4,8 and 12 by calculating.

- Proportion of patients achieving a $\geq 75 \%$ or $>90 \%$ reduction in mPASI

- Change from baseline in BSA affected

- Proportion of patients who were clear/almost clear of psoriasis, with a $\geq 2$ grade improvement according to PGA (defined as treatment success') - Patients completed the DLOI questionnaire at baseline and weeks 4, 8 and 12 (range 0-30). Quality of life was assessed by calculating the proportion of patients achieving:

- DLOI score of 0/1 (ie no impact of psoriasis on the patient's life) - Decrease in DLQI score of $\geq 5$ (ie the minimal clinically important difference) - The amount of each product used throughout the study was also assessed STATISTICAL ANALYSIS

- Analyses were conducted on the full analysis set, which comprised all patients ate-to-severe psoriasis - Last observation carried forward (LOCF) was used to impute values for missing
mPASI data. An observed case approach was used for other variables
Results

PATIENTS

463 patients were randomized to Cal/BD foam ( $(n=185)$, Cal/BD gel

( $n=188)$, foam vehicle $(n=47)$ and gel vehicle $(n=43)$

- Seventy-seven Cal/BD foam patients and $82 \mathrm{Cal} / \mathrm{BD}$ gel patients

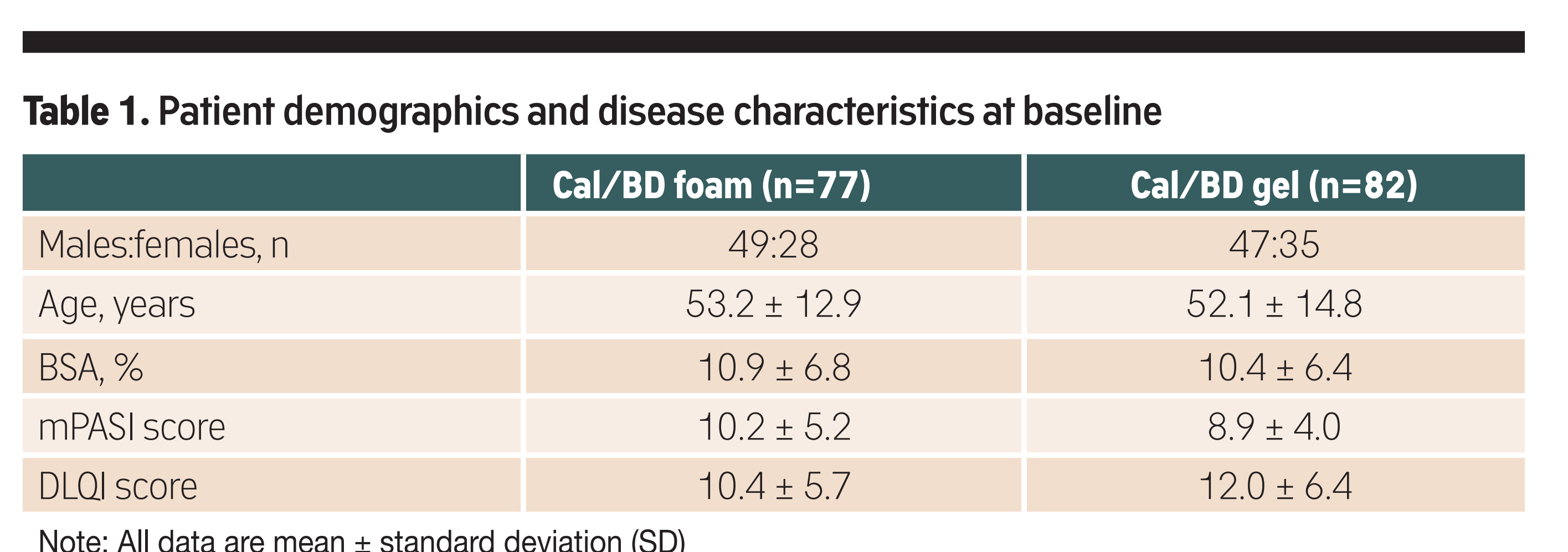

MPASI SCORES

The proportion of patients achieving mPASI75 and mPASI90 was greater with

Cal/BD foam than Cal/BD gel at weeks 4, 8 and 12 (Figure 1)

- Percentage mean ( \pm SD) reduction in mPASI from baseline to week 12 was
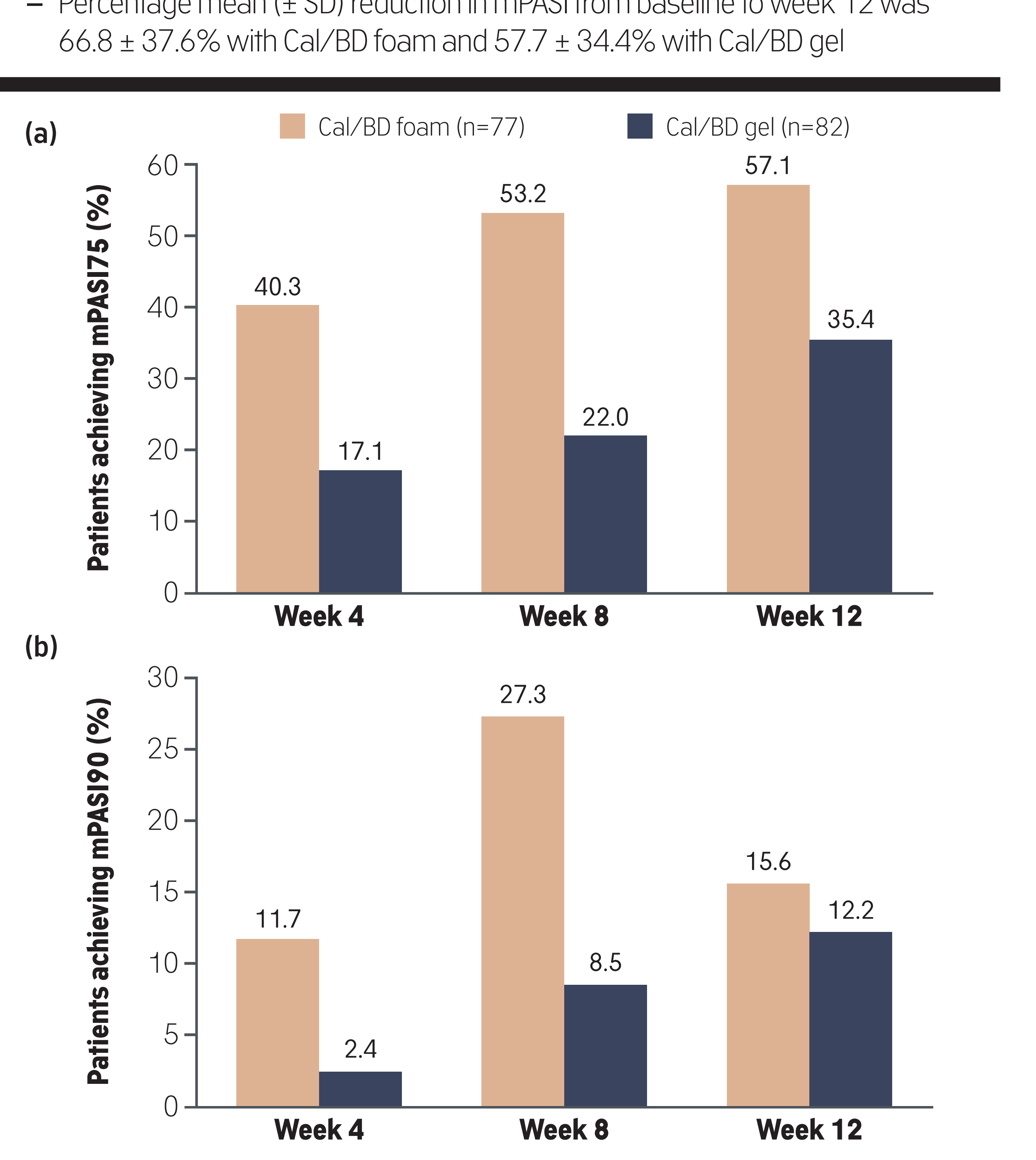

Figure 1. Proportion of patients with moderate-to-severe psoriasis achieving (a) mPAS175

\section{DSA AFFECTED BY PSORIASIS}

- The proportion of BSA affected decreased
foam and Cal/BD gel groups (Figure 2)

Percentage mean $( \pm \mathrm{SD}$ ) reduction from baseline to week
$43.0 \%$ with Cal/ BD foam and $39.2 \pm 37.7 \%$ for Cal/BD ge

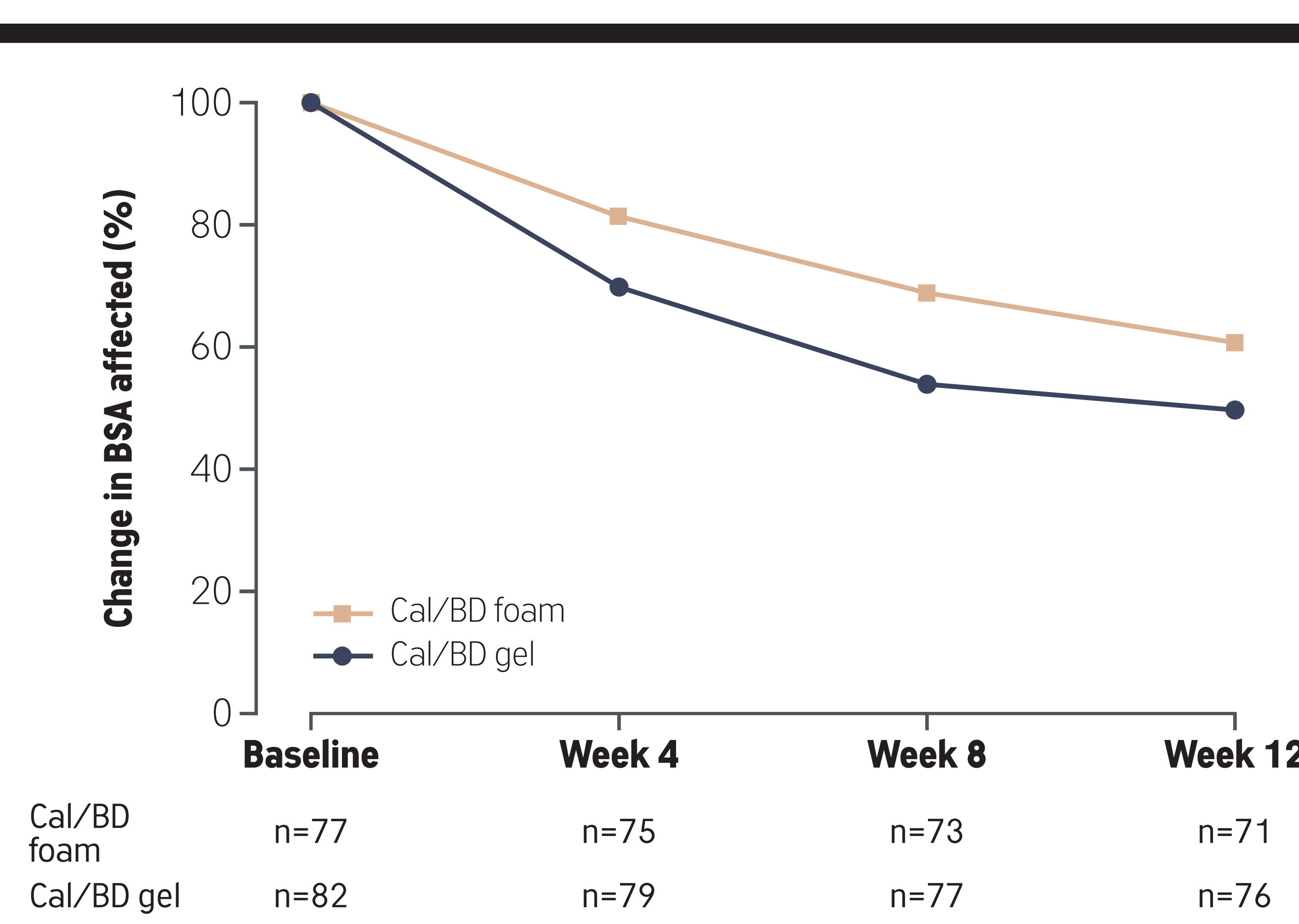

Figure 2. Reduction in BSA affected by psoriasis from baseline in moderate--to-severe

TREATMENT SUCCESS

- Treatment success rates increased throughout the first 6 weeks, reaching

increase up to week 12 (Figure 3 )

- Success rates were higher with Cal/BD foam than Cal/BD gel at

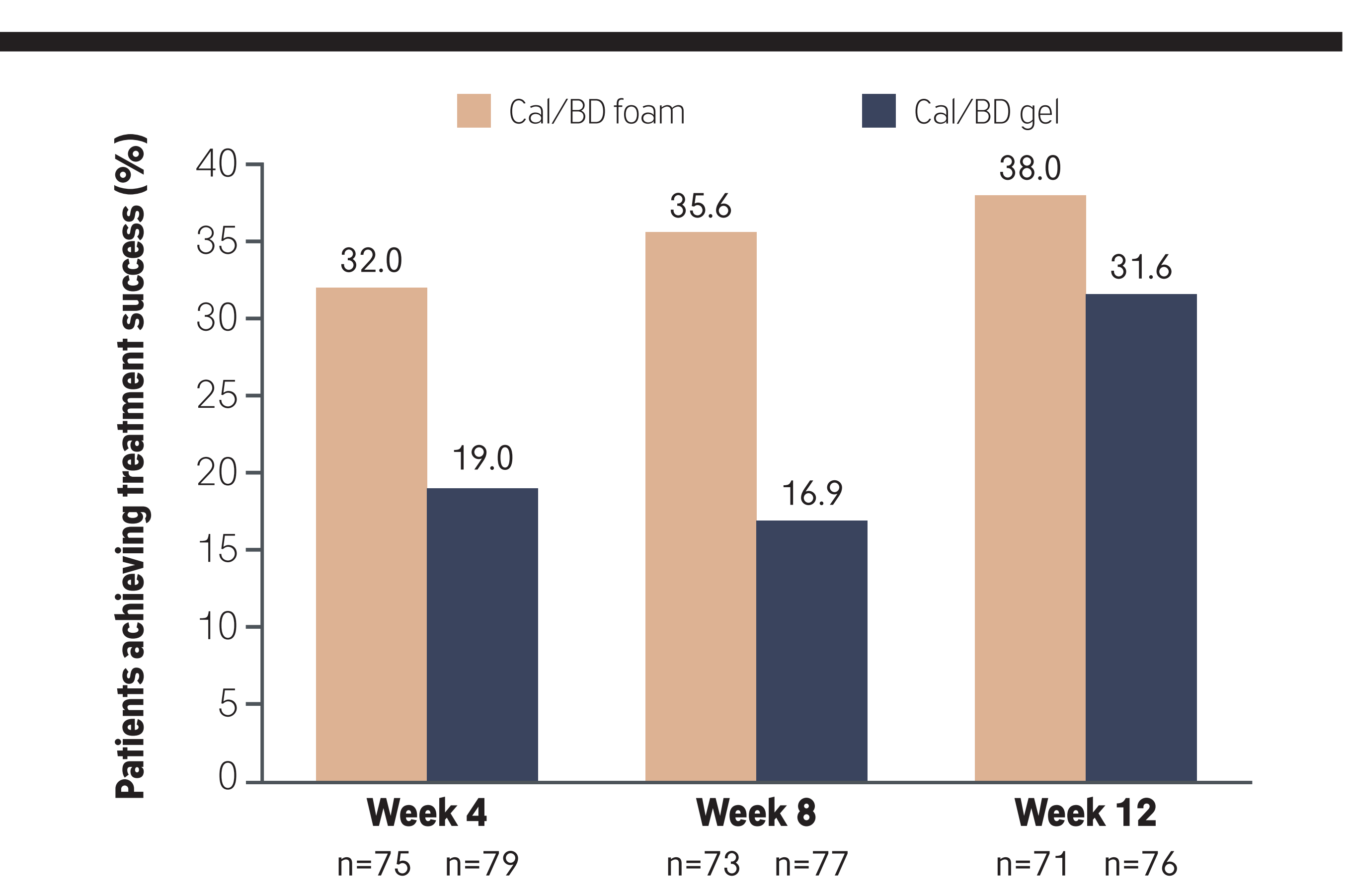

Figure 3. Proportion of mode

DLOI SCORES

- A greater proportion of patients achieved a DLQ1 of 0/1 at weeks 4, 8 and 12 with Cal/BD foam than Cal/BD gel (Figure 4)

- The proportion of patients achieving a decrease in DLOl of $\geq 5$ with Cal/BD foam was greater than with Cal/BD gel at week $4(70.3 \%$ vs $56.4 \%)$,
then similar at weeks $8(68.5 \%$ vs $66.2 \%$ and $12(62.9 \%$ vs $64.0 \%)$

AMOUNT OF PRODUCT USED

The mean amount of Cal/BD foam used was $28.0 \pm 20.3 \mathrm{~g} /$ week

The greatest usage of Cal/BD foam occurred in the first 6 weeks

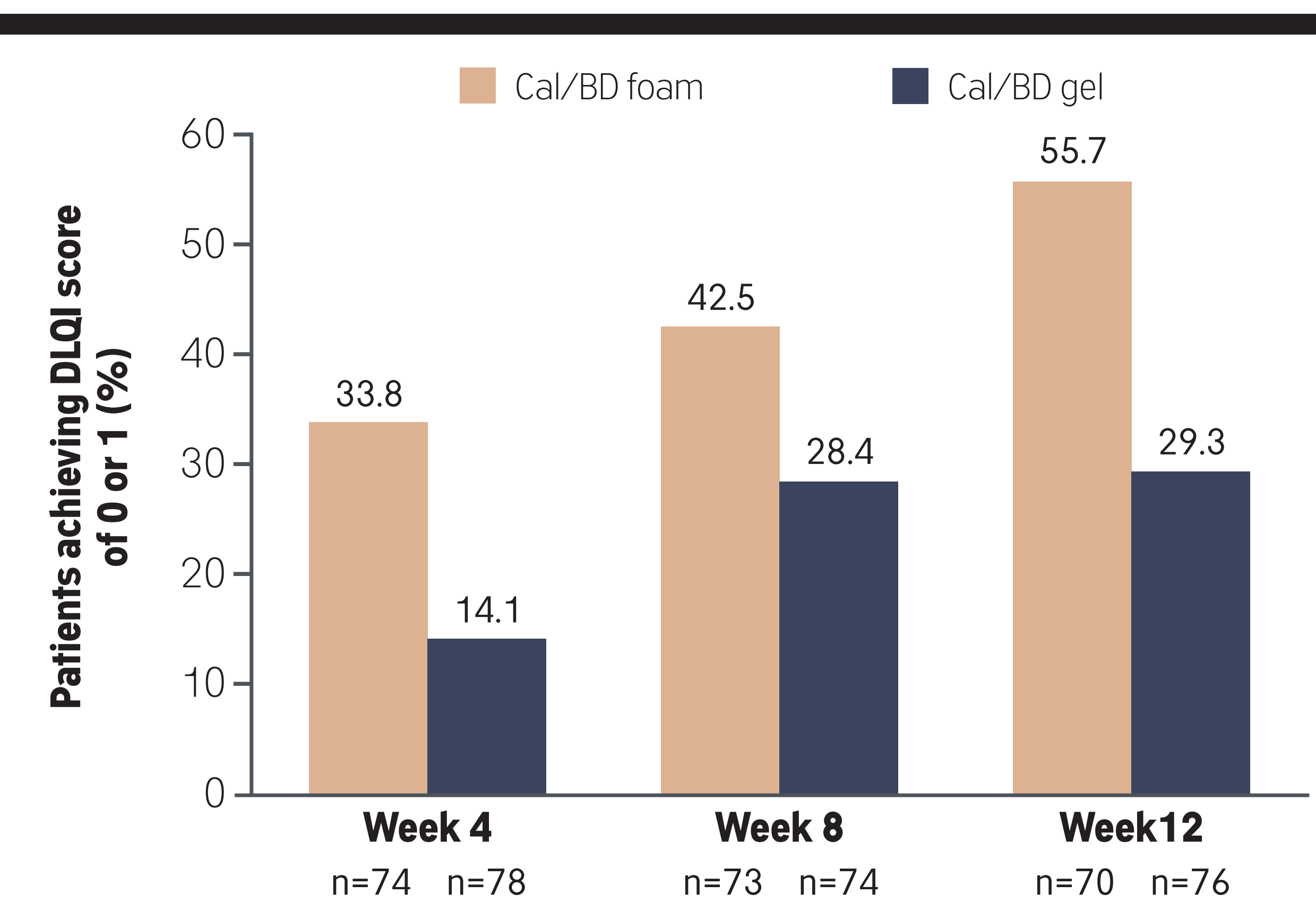

Figure 4. Proportion of patients achieving a DLOI score of $0 / 1$ ( (bbserved cases)

Conclusions

\begin{abstract}
This subgroup analysis demonstrates that Cal/BD foam is effective in patients with moderate-to-severe psoriasis; it should be noted however, that it is difficult to treat psoriasis patients who have large BSA involvement purely with topical therapy. The superior efficacy of Cal/BD foam over Cal/BD gel that was observed in the primary PSO-ABLE study ${ }^{8}$ was maintained for up to 12 weeks in these patients

Potential limitations of this analysis: the definition of moderate-to-severe (based on the "Rule of Tens") differs from the used in studies of systemic therapies, where patients a typically required to have BSA $\geq 10 \%$ and $\mathrm{PASI}>10$; mean to 10 , therefore on the threshold for moderate-to-severe psoriasis

This subanalysis suggests Cal/BD foam may be a costsaving alternative to systemic therapies, in some patients with moderate-to-severe psoriasis who are able to maintain adherence to topical therapy and do not want to be exposed to systemic therapy
\end{abstract}

Acknowledgements

This studdy was s sononsored by LEO Pharma. Medical writing support was provided b

References

. Menter A et al. J Am Acad Dermatol 2009;60:643-65

Nast Aet al. Arch Dermatol Res 2012;304:87-113.

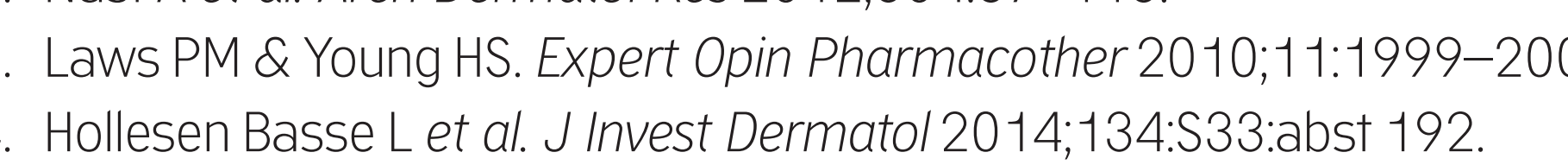

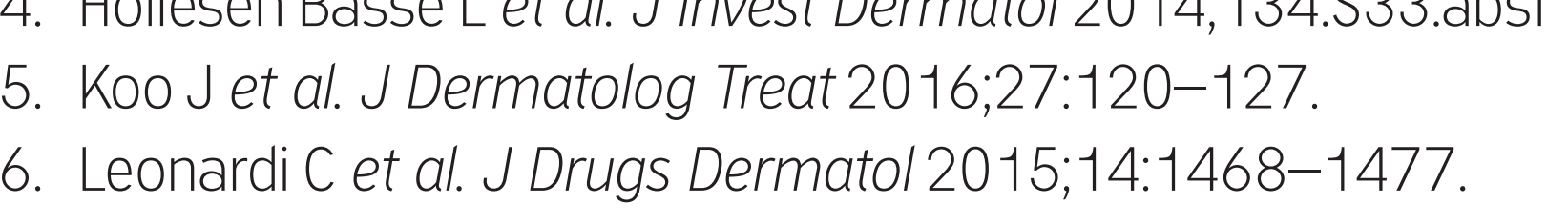

Q Quelle-Roussel Cetal. Clin Drug Investig 2015,35:239-24. 